\title{
SPECTRA OF VARIABLE WHITE DWARFS
}

\author{
Jesse L. Greenstein \\ Hale Observatories \\ California Institute of Technology
}

Spectra of seven ZZ Cet1 DA variables have been obtained with the multichannel spectrophotometer, several also with $7 \AA$ resolution with the SIT digital spectrophotometer, one with the IUE. The colors fit hydrogen model atmospheres well; the combined IUE and MCSP energy distribution of G29-38 show no pecullarity from 10,000 to $2000 \AA$. Hydrogen model atmospheres yleld $\langle\log \mathrm{T}\rangle=4.073 \pm 0.008$; the dispersion is $\sigma= \pm 0.02$, quite near the total observational and model-fitting error. Using an estimate of the intrinsic $2 \sigma$ as a half-width the instability strip for hydrogen degenerates 1 les at $11,800 \pm 600 \mathrm{~K}$ (for $\log \mathrm{g}=8$ ). This applies, of course, only to the northern $2 Z$ Ceti stars observed. Another measure of how normal these variables appear is the equivalent width of Balmer lines, which are normal for that $T$, and are at the line-strength maximum (estimated for $\log g=8.2$ ). Possibly the mean $H \alpha$ is slightly strong; since the Balmer jump is also at maximum strength, a slightly anomalous low boundary temperature might be present. This is also suggested by marginally more positive U-B color for their G-R (or larger log $g$ ). I have counts from MCSP data giving the frequency of $162 \mathrm{DA}$ effective temperatures. The $\mathrm{ZZ}$ Cet1 stars represent 7 of 27 stars in the bin \pm 0.05 wide in $\log T$ centered on 4.05. They are a substantial fraction, but not necessarily all, stars at that temperature. An attempt to observe $\mathrm{H \alpha}$ emission with the Boksenberg IPCS at the Palomar coudé falled for lack of photons.

From observations with IUE, by Oke and myself, the AM CVn (HZ 29) spectrum is quite different; the predicted flux in a helium atmosphere, followed into the ultraviolet, is replaced by a nearly flat $\left(f_{v}=\right.$ constant) on which are superposed strong $P$ Cygni, violet displaced deep absorption lines, N V, He II, C IV, SI IV, SI III. Geocoronal Lyman emission hides any possible weak hydrogen-shell absorption. Outflow velocities are near $-600 \mathrm{~km} \mathrm{~s}^{-1}$, in an outer reglon of hot (50 eV), expanding hydrogen-poor gas, absorbing the light elther of a hellumaccretion disk or of a helium degenerate model. At this temperature, photometry does not clearly decide whether the ultraviolet is nonthermal. But in Iine spectrum it resembles the hydrogen-rich envelope of the erratic variable $-7^{\circ} 3007$ except for $L \alpha^{\prime} s$ absence. The apparent doubling of the optical-region He I absorption lines of HZ 29 (see Faulkner) may result from weak hellum recombination in the outer envelope. Both stars in $\mathrm{AM} \mathrm{CVn}$ are helium rich; the object undergoing 
mass loss has metals also. The star's effective temperature is too low to lonize $\mathrm{N}, \mathrm{C}, \mathrm{S} 1$ to the level found. If the flat ultraviolet continuum is that of a disk we are close to the model suggested by Faulkner et al. (1972, 1975).

\section{REFERENCES}

Faulkner, J., and Robinson, E. L. 1975, Ap。 Jo (Letters), 200, L23. Faulkner, J., Flannery, B. P., and Warner, B. 1972, Ap。J. (Letters), 175, L79.

McGraw, J. T. 1979, these Proceedings. 